\title{
Significance of KDM6A mutation in bladder cancer immune escape
}

\author{
Xingxing Chen, Xuehua Lin, Guofu Pang, Jian Deng, Qun Xie and Zhengrong Zhang*
}

\begin{abstract}
Background: Bladder cancer (BC) is the fourth most prevalent neoplasm in men and is associated with high tumour recurrence rates, leading to major treatment challenges. Lysine-specific demethylase 6A (KDM6A) is frequently mutated in several cancer types; however, its effects on tumour progression and clinical outcome in $B C$ remain unclear. Here, we explored the potential role of KDM6A in regulating the antitumor immune response.

Methods: We mined The Cancer Genome Atlas (TCGA) and International Cancer Genome Consortium (ICGC) databases for somatic mutation and clinical data in patients with BC.

Results: We found frequent mutations in 12 genes in both cohorts, including TP53, KDM6A, CSMD3, MUC16, STAG2, PIK3CA, ARID1A, RB1, EP300, ERBB22, ERBB3, and FGFR3. The frequency o KDM6A mutations in the TCGA and ICGC datasets was 25.97 and $24.27 \%$, respectively. In addition, KDM6A mutation was associated with a lower number of tumour-infiltrating immune cells (TIICS) and indicated a state of immune tolerance. KDM6A mutation was associated with lower KDM6A mRNA level compared with that in samples carrying the wild-type gene. Further, survival analysis showed that the prognosis of patients with low KDM6A expression was worse than that with high KDM6A expression. Using the CIBERSORT algorithm, Tumor Immune Estimation Resource site, and Gene Set Enrichment Analysis, we found that KDM6A mutation downregulated nine signalling pathways that participate in the immune system and attenuated the tumour immune response.
\end{abstract}

Conclusion: Overall, we conclude that KDM6A mutation is frequent in $B C$ and promotes tumour immune escape, which may serve as a novel biomarker to predict the immune response.

Keywords: KDM6A, Bladder cancer, Mutation, Immune

\section{Introduction}

Bladder cancer $(\mathrm{BC})$ is considered to be the fourth most prevalent neoplasm in men [1]. BC is associated with high rates of tumour recurrence and progression, which represent major challenges in treatment. Lysine-specific demethylase 6A (KDM6A) is a member of the histone H3 lysine 27 demethylase gene family, which is reported to exert pro-tumorigenic effects in some cancer types [2, 3] but is also considered a tumour suppressor in other contexts [4]. Analyses of tumour-associated genes

\footnotetext{
* Correspondence: 76240500@qq.com

Department of Urology, Zhuhai People's Hospital, Zhuhai Hospital affiliated with Jinan University, Kangning Road No.79, in Zhuhai city, Guang Dong Province, Zhuhai, People's Republic of China
}

showed frequent loss of $K D M 6 A$ expression in several cancers, including BC, B-cell lymphoma, lung squamous cell carcinoma, head and neck squamous cell carcinoma, pancreatic adenocarcinoma, and renal papillary cell carcinoma [5-9]. Nevertheless, the molecular mechanism by which $K D M 6 A$ might suppress tumour progression in $\mathrm{BC}$ remains unclear. To provide insight into this mechanism, we conducted data mining of two separate public cohorts of $\mathrm{BC}$ patients to explore the frequency of $K D M 6 A$ mutations and its potential influence on the immune response. This study can help to elucidate the effect of KDM6A on the tumour microenvironment and develop a potential immunotherapy strategy for $\mathrm{BC}$ patients.

(c) The Author(s). 2021 Open Access This article is licensed under a Creative Commons Attribution 4.0 International License, which permits use, sharing, adaptation, distribution and reproduction in any medium or format, as long as you give appropriate credit to the original author(s) and the source, provide a link to the Creative Commons licence, and indicate if changes were made. The images or other third party material in this article are included in the article's Creative Commons licence, unless indicated otherwise in a credit line to the material. If material is not included in the article's Creative Commons licence and your intended use is not permitted by statutory regulation or exceeds the permitted use, you will need to obtain permission directly from the copyright holder. To view a copy of this licence, visit http://creativecommons.org/licenses/by/4.0/ The Creative Commons Public Domain Dedication waiver (http://creativecommons.org/publicdomain/zero/1.0/) applies to the data made available in this article, unless otherwise stated in a credit line to the data. 


\section{Methods}

\section{Datasets}

The somatic gene mutations and clinical data of 412 samples from BC patients in the United States and 101 samples from BC patients in China were downloaded from The Cancer Genome Atlas (TCGA) and International Cancer Genome Consortium (ICGC) online platforms, respectively. Tumor immune estimation resource downloaded from TIMER2.0 (http://timer. cistrome.org/).

\section{Data analysis}

All statistical analyses were performed using Stata software 14.0 and GraphPad Prism 8.0 (San Diego, CA, USA); $P<0.05$ was defined as statistically significant. Kaplan-Meier survival analysis was conducted to generate survival curves, which were evaluated using the log-rank test. Cox regression analyses were performed for assessing the associations of survival with clinical characteristics and KDM6A expression levels. The Kruskal-Wallis test was used to analyse the correlation between $K D M 6 A$ mutation status and overall mutation counts.

CIBERSORT was conducted to evaluate the proportions of 22 tumor-infiltrating immune cells (TIICs) subsets in 412 samples from BC patients in the United States, and estimated the relative abundance of TIICs with different KDM6Astatus.

Gene set enrichment analysis (GSEA) was performed using GSEA 4.0. Student's $t$-test was used to compare the expression level of $K D M 6 A$ mRNA according to the $K D M 6 A$ mutation status. The $\mathrm{X}^{2}$ test was used to evaluate the association between KDM6A mutation status and clinical parameters. In GSEA, $P<0.05$ and $\mathrm{q}<0.25$ were considered statistically significant.

\section{Results}

\section{KDM6A mutation is frequent in $\mathrm{BC}$}

We downloaded the data, including follow-up profiles and gene expression levels, of 411 and 103 BC tissues from the TCGA and ICGC databases, respectively. Among these samples, $107 / 411$ (25.97\%) and 25/103 (24.27\%) harboured a KDM6A mutation (Sup. 1A-C).

Missense and truncating mutations were the main mutation types spanning the entire gene (Sup.2A-B), with the former mutation type being most frequent (Sup. 3A); single nucleotide polymorphism was more common than deletion or insertion (Sup. 3B), and $\mathrm{C}>\mathrm{T}$ was the most common single nucleotide variant in BC (Sup. 3C). The number of variant bases in each sample was counted and the mutation types are shown in a box plot in different colours in Sup. 3D and 3E.
KDM6A mutation is associated with higher mutation counts

BC samples with $K D M 6 A$ mutations had higher overall mutation counts than wild-type samples in the TCGA cohort (median mutation counts: 210 vs 156.5; $p=$ 0.0383 , Kruskal-Wallis equality-of-populations rank test) (Fig. 1).

\section{$K D M 6 A$ mutation is associated with reduced $K D M 6 A$ mRNA levels and poor prognosis in BC}

Patients with KDM6A mutations had lower KDM6A mRNA levels than those with wild-type $K D M 6 A$ (Fig. 2A). As shown in Sup.4, patients with KDM6A mutations had a higher $M$ stage (TNM staging) than those with wild-type KDM6A. However, no correlation was observed between $K D M 6 A$ mutation and age, histologic grade, or the tumour-node-metastasis (TNM) stage. Kaplan-Meier analysis showed that $\mathrm{BC}$ patients with a high $K D M 6 A$ mRNA (top $15 \%, n=61$ ) levels had significantly longer overall survival than those with low $K D M 6 A$ levels (bottom 15\%, $n=61$ ) (Fig. 2B). However, univariate and multivariate Cox regression analyses showed that a low KDM6A level or presence of $K D M 6 A$ mutation was not an independent prognostic factor.

$K D M 6 A$ mutation is significantly correlated with tumourinfiltrating immune cells (TIICS) in the tumour microenvironment

Tumor Immune Estimation Resource (TIMER) analysis showed that KDM6A mutation was associated with a lower number of TIICs. The infiltration levels of macrophages $(p=1.44 \mathrm{e}-2)$, CD8+ T cells $(p=5.79 \mathrm{e}-4)$, neutrophils $(p=4.40 \mathrm{e}-3)$, and resting dendritic cells $(p=3.70 \mathrm{e}-$ 3 ) in the KDM6A mutation group were lower than those in the $K D M 6 A$ wild-type group (Fig. $3 \mathrm{~A}$ ). The association between KDM6A mutation and different types of immune cells was significant, including dendritic cells $(p=9.70 \mathrm{e}-3$, cor $=-0.1272)$, CD8 T cells $(p=6.0 \mathrm{e}-4$, cor $=-0.1689), \quad$ macrophages $\quad(p=1.03 \mathrm{e}-2, \quad$ cor $=-$ $0.1262)$, and neutrophils ( $p=5.1 \mathrm{e}-3$, cor $=-0.1375)$.

To further assess the correlation of KDM6A mutation with TIICs in the BC microenvironment, the CIBERSORT algorithm was used. The composition of 22 TIICs in each sample varied significantly (Fig. 3B). Moreover, naive $\mathrm{B}$ cells were more enriched in the KDM6A mutation group, whereas M2 macrophages and resting mast cells were more enriched in the KDM6A wild-type group (Fig. 3C). Correlation analysis showed that memoryactivated CD4+ $\mathrm{T}$ cells were positively correlated with CD8+ $\mathrm{T}$ cells, and also had a positive association with the number of M1 macrophages. In turn, resting macrophages were positively correlated with levels of activated natural killer (NK) cells. However, activated 


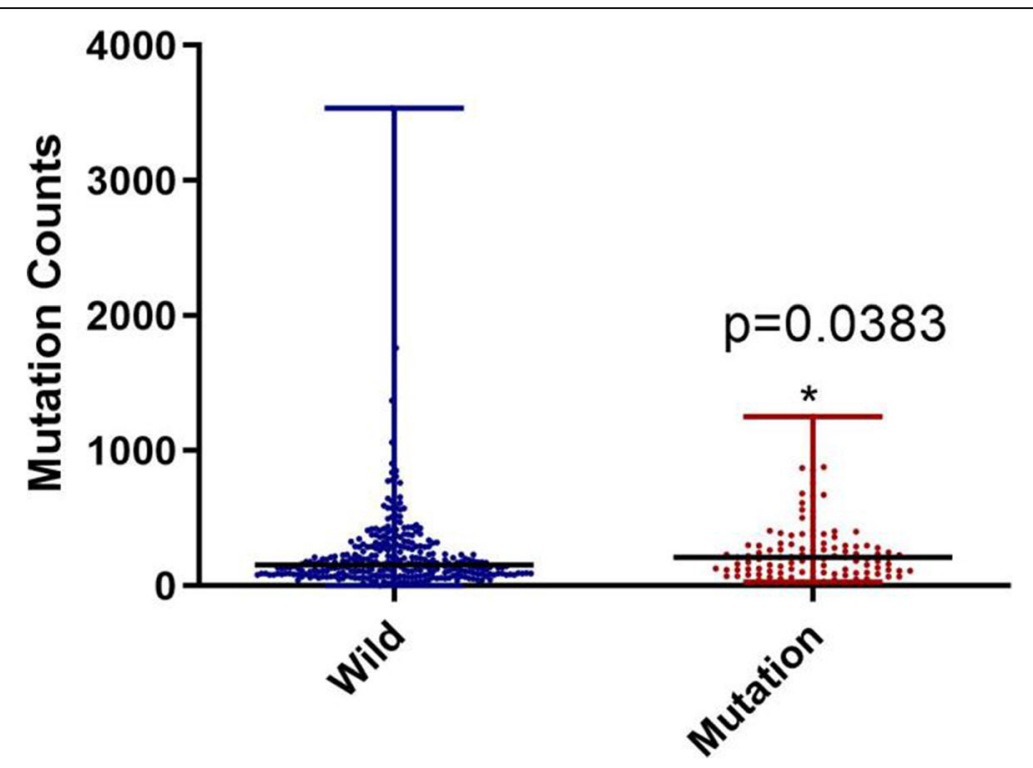

Fig. $1 \mathrm{KDM} 6 \mathrm{~A}$ mutation was associated with mutation counts. KDM6A mutation was associated with a higher mutation counts

NK cells were negatively correlated with activated mast cells (Fig. 3D).

\section{Enrichment pathway analysis of KDM6A mutation}

We further explored the relationship between $K D M 6 A$ mutation and the immune response. GSEA revealed that the intestinal immune network for IgA production, the chemokine signalling pathway, natural killer cell-mediated cytotoxicity, the $\mathrm{B}$ cell receptor signalling pathway, the $\mathrm{T}$ cell receptor signalling pathway, the Fc epsilon Ri signalling pathway, Fc gamma R-mediated phagocytosis, primary immunodeficiency, and the Toll-like receptor signal pathway was significantly downregulated in the $K D M 6 A$ mutation group (Fig. 4A-I). These results implied that KDM6A mutation downregulates signalling pathways involved in the immune system in $\mathrm{BC}$.

\section{Discussion}

Dysfunction in demethylation occurs frequently in cancer cells, which can destroy the chromatin configuration and disrupt normal transcriptional processes. KDM6A is a specific H3K27me3 demethylase, and inactivating mutations in $K D M 6 A$ have been frequently detected in $\mathrm{BC}$ [1]. $K D M 6 A$ was suggested to act as a tumour suppressor in

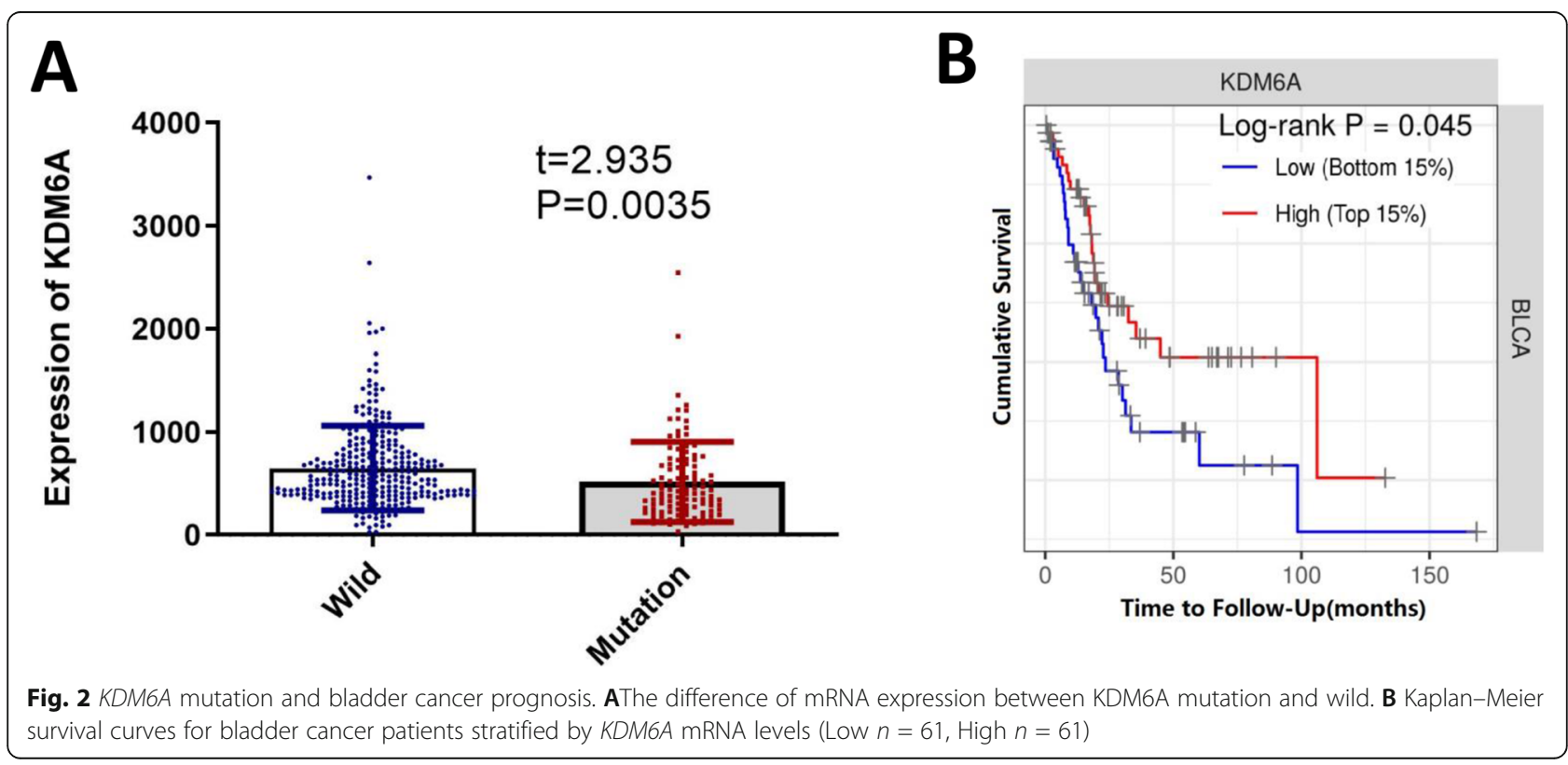




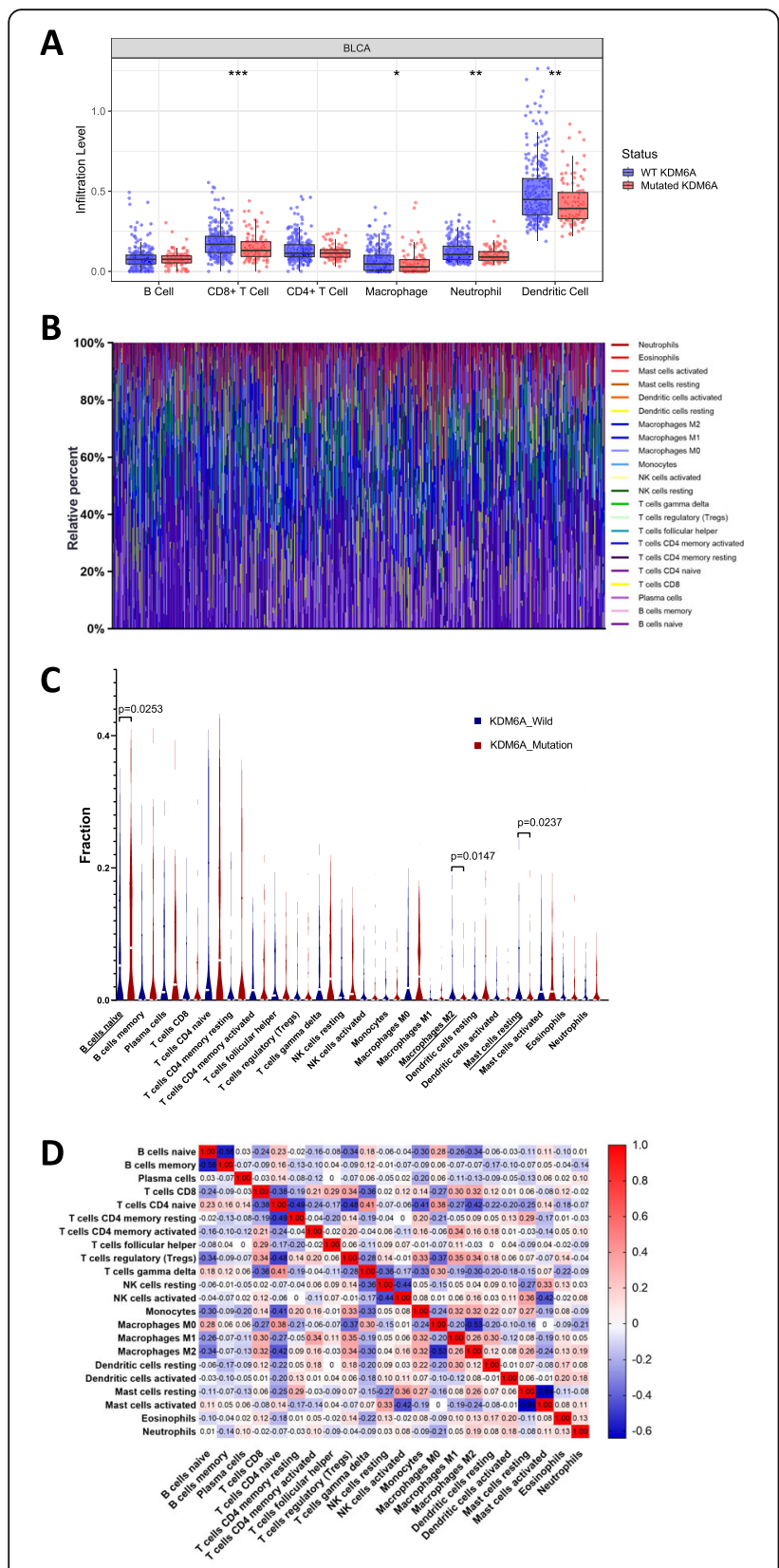

Fig. 3 KDM6A mutation was correlated with tumor-infiltrating immune cells. A The diffrence of 8 immune cells between KDM6A mutation group and KDM6A wild-type group. Blue color represented $K D M 6 A$ wild-type group, and red color represents KDM6A mutation group. ${ }^{*} p<0.05 ;{ }^{* *} p<0.01 ;{ }^{* *} p<0.001$ B Stacked bar chart showed distribution of 22 immune cells in each sample. $\mathbf{C}$ Violin plot displayed the differentially infiltrated immune cells between KDM6A mutation group and KDM6A wild-type group. Blue color represented KDM6A wild-type group, and red color represented KDM6A mutation group. D Correlation matrix of immune cell proportions. The red color represented positive correlation and the blue color represented negative correlation several cancers [10]. Other studies reported abnormal levels of trimethylated H3K27 (H3K27me3) in some cancers, which correlated with a poor prognosis [11-13], suggesting that demethylases are involved in both oncogenesis and tumour progression. Several recent studies identified inactivating mutations of $K D M 6 A$ in different cancer tissues, along with decreased expression levels of $K D M 6 A$ in cancer compared with those in normal tissues, further supporting that $K D M 6 A$ is a tumour suppressor [14-17]. KDM6A mutation is common especially in women with $B C[18,19]$. Mutations in or a decreased expression level of $K D M 6 A$ was also associated with a poor prognosis of female BC patients [20].

Our present analysis of the somatic mutation landscape data of BC samples from the TCGA dataset (412 cases) and ICGC dataset (101 cases) confirmed that $K D M 6 A$ was frequently mutated in both cohorts, in line with previous studies $[6,19]$. Moreover, KDM6A mutation was correlated with higher overall gene mutation counts, but was not associated with clinical prognosis.

Importantly, we identified that KDM6A mutation in $\mathrm{BC}$ was negatively associated with signalling pathways involved in the immune response. We observed reduced infiltration of immune cells in KDM6A-mutated tissues, including neutrophils, macrophages, and CD8+ T cells, which is consistent with previous reports showing that these immune cells play key roles in the tumour microenvironment and suppress the immune response. A reduced number of CD8+ T cells in the tumour indicated a worse prognosis for patients in a previous study [21]. GSEA of Kyoto Encyclopedia of Genes and Genomes pathways between gene sets of $\mathrm{BC}$ samples with mutated and wild-type $K D M 6 A$ demonstrated that the signalling pathways of cell-adhesion molecules, ECM receptor interaction, and focal adhesion were also suppressed by KDM6A mutation. These findings support the need for further in-depth study of the possible role of $K D M 6 A$ in regulation of cell adhesion and morphology in $B C$ [22]. $K D M 6 A$ deficiency has been suggested to activate pathways of chemokines and cytokines, increase M2 macrophage polarization, increase cancer stem cell abundance, and act synergistically with p53 haploinsufficiency [23]. Given the key function of KDM6A in regulating CD4+ $\mathrm{T}$ cells [24], concluded that KDM6A likely regulates multiple immune response genes in autoimmune disease susceptibility.

There are several limitations in this study. Fist, specimens of superficial bladder cancer are a few. Therefore, more studies could conduct on invasive bladder cancer and superficial bladder cancer, separately. Second, the molecular mechanism by which KDM6A might suppress tumour progression need further research. Third, although our results suggested that BC patients with a high KDM6A mRNA (top 


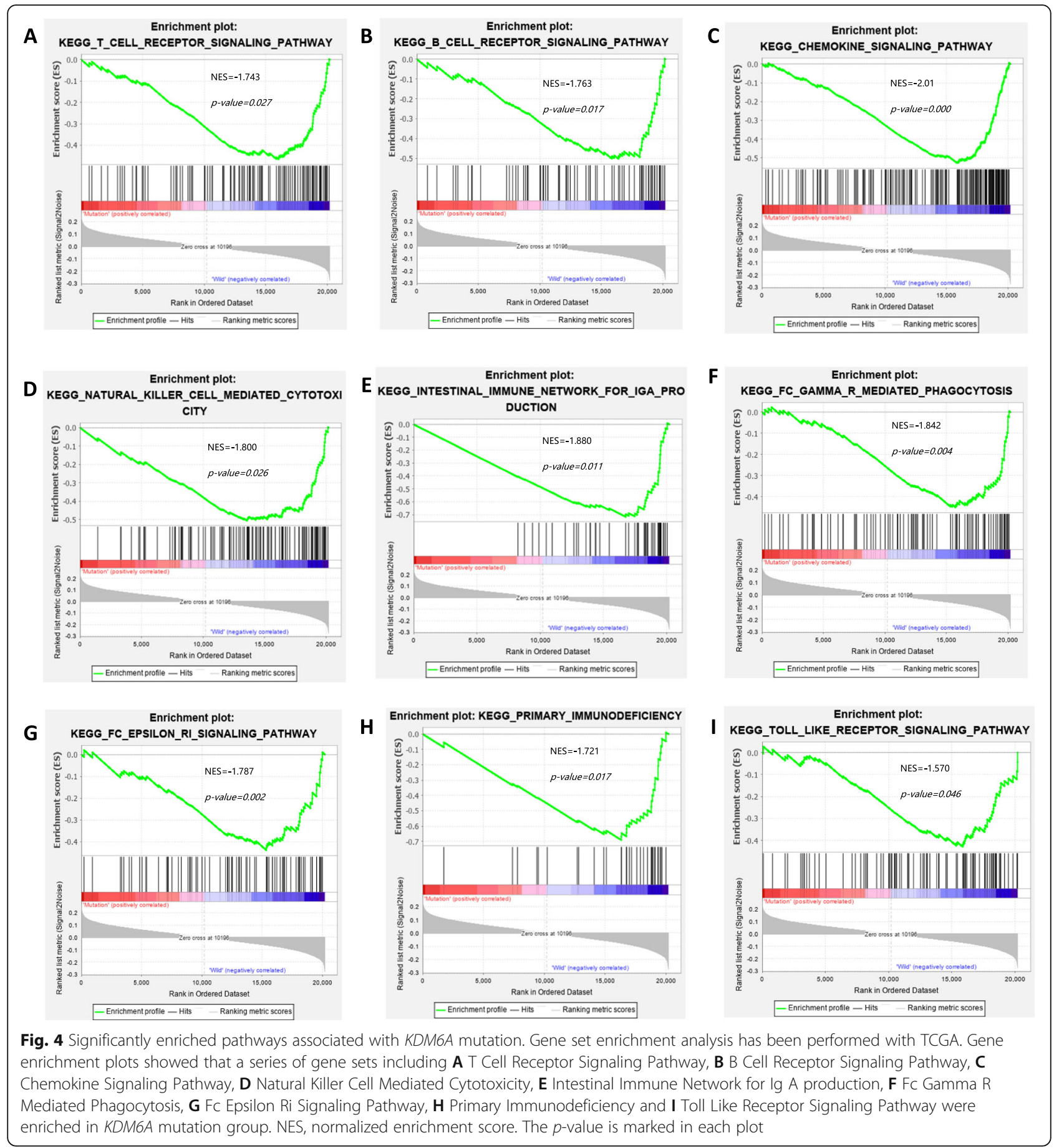

$15 \%, n=61$ ) levels had significantly longer overall survival than those with low KDM6A levels (bottom $15 \%, n=61)$, but there is insufficient high-quality data and more studies are needed. Finally, although our results showed naive $\mathrm{B}$ cells were more enriched in the KDM6A mutation group, it is still unclear whether naive $B$ cells are involved in immune escape and the mechanism, further study is needed.

\section{Conclusions}

This study identified that $K D M 6 A$ was frequently mutated in $\mathrm{BC}$, and $K D M 6 A$ mutation was correlated with a higher overall gene mutation status. Furthermore, KDM6A mutation was shown to negatively regulate the signalling pathways of the immune system and to suppress antitumor immunity. These findings suggest KDM6A as a latent gene 
whose mutation could be considered as a predictive biomarker for the immune response, thereby facilitating the development of new strategies for immunotherapy and treatment monitoring in $\mathrm{BC}$ patients.

\section{Abbreviations}

KDM6A: Lysine-specific demethylase 6A; BC: Bladder cancer; TCGA: The Cancer Genome Atlas; ICGC: International Cancer Genome Consortium; GSEA: Gene Set Enrichment Analysis; TNM: Tumour-node-metastasis; TIICs: Tumour-infiltrating immune cells; TIMER: Tumor immune estimation resource.; TP53: Tumor protein p53; CSMD3: CUB And Sushi Multiple Domains 3; MUC16: Mucin 16; STAG2: Stromal antigen2; PIK3CA: Phosphoinositide-3kinase, catalytic, alpha; ARID1A: AT-rich interactive domain containing protein 1A; RB1: Retinoblastoma1; EP300: E1A binding protein p300; ERBB2: Erb-b2 receptor tyrosine kinase 2; ERBB3: Erb-b2 receptor tyrosine kinase 2; FGFR3: Fibroblast growth factor receptor 3; NK cells: Natural killer cells

\section{Supplementary Information}

The online version contains supplementary material available at https://doi. org/10.1186/s12885-021-08372-9.

\section{Additional file 1.}

\section{Acknowledgements}

Not applicable.

\section{Authors' contributions}

Chen X., Lin X. and Zhang Z. conceived of the study, participated in its design and coordinated and drafted the manuscript. Chen $X$. and Lin $X$. performed the statistical analyses. Pang G., Deng J., Xie Q. and Zhang Z. gave critical advice and participated in manuscript revising. All authors read and approved the final manuscript.

\section{Funding}

None.

\section{Availability of data and materials}

The datasets used during the present study are available from the Corresponding author upon reasonable request. Data were obtained from The Cancer Genome Atlas (TCGA; https://portal.gdc.cancer.gov), International Cancer Genome Consortium (ICGC; https://dcc.icgc.org/) and TIMER (http:// timer.cistrome.org/)

\section{Declarations}

\section{Competing of interests}

None declared.

\section{Ethics approval and consent to participate}

The data is publicly available therefore no Ethics approval is required.

\section{Consent for publication}

Not applicable.

Received: 23 November 2020 Accepted: 6 May 2021

Published online: 29 May 2021

\section{References}

1. Qiu JG, Shi DY, Liu X, Zheng XX, Wang L, Li Q. Chromatin-regulatory genes served as potential therapeutic targets for patients with urothelial bladder carcinoma. J Cell Physiol. 2019;234(5):6976-82. https:// doi.org/10.1002/jcp.27440.

2. Schulz WA, Lang A, Koch J, Greife A. The histone demethylase UTX/KDM6A in cancer: Progress and puzzles. Int J Cancer. 2019;145(3):614-20. https://doi. org/10.1002/ijc.32116.

3. Wang L, Shilatifard A. UTX mutations in human Cancer. Cancer Cell. 2019; 35(2):168-76. https://doi.org/10.1016/j.ccell.2019.01.001.
4. Van der Meulen J, Speleman F, Van Vlierberghe P. The H3K27me3 demethylase UTX in normal development and disease. Epigenetics. 2014; 9(5):658-68. https://doi.org/10.4161/epi.28298.

5. Kandoth C, McLellan MD, Vandin F, Ye K, Niu B, Lu C, et al. Mutational landscape and significance across 12 major cancer types. Nature. 2013; 502(7471):333-9. https://doi.org/10.1038/nature12634.

6. Chang S, Yim S, Park H. The cancer driver genes IDH1/2, JARID1C/ KDM5C, and UTX/ KDM6A: crosstalk between histone demethylation and hypoxic reprogramming in cancer metabolism. Exp Mol Med. 2019;51(6):1-17. https://doi.org/10.1038/s12276-019-0230-6.

7. Li X, Zhang Y, Zheng L, Liu M, Chen CD, Jiang H. UTX is an escape from Xinactivation tumor-suppressor in B cell lymphoma. Nat Commun. 2018;9(1): 2720. https://doi.org/10.1038/s41467-018-05084-w.

8. Linehan WM, Spellman PT, Ricketts CJ, Creighton CJ, Fei SS, Davis C, et al. Comprehensive molecular characterization of papillary renal-cell carcinoma. N Engl J Med. 2016;374(2):135-45.

9. Liu J, Lee W, Jiang Z, Chen Z, Jhunjhunwala S, Haverty PM, et al. Genome and transcriptome sequencing of lung cancers reveal diverse mutational and splicing events. Genome Res. 2012;22(12):2315-27. https://doi.org/10.1101/gr.140988.112.

10. Kim JH, Sharma A, Dhar SS, Lee SH, Gu B, Chan CH, et al. UTX and MLL4 coordinately regulate transcriptional programs for cell proliferation and invasiveness in breast cancer cells. Cancer Res. 2014;74(6):1705-17. https:// doi.org/10.1158/0008-5472.CAN-13-1896

11. Wei Y, Xia W, Zhang Z, Liu J, Wang H, Adsay NV, et al. Loss of trimethylation at lysine 27 of histone $\mathrm{H} 3$ is a predictor of poor outcome in breast, ovarian, and pancreatic cancers. Mol Carcinog. 2008:47(9):701-6. https://doi.org/10.1002/mc20413.

12. Pellakuru LG, Iwata T, Gurel B, Schultz D, Hicks J, Bethel C, et al. Global levels of H3K27me3 track with differentiation in vivo and are deregulated by MYC in prostate cancer. Am J Pathol. 2012;181(2):560-9. https:/doi.org/10.1016/j.ajpath.2012.04.021.

13. Sun S, Yang Q, Cai E, Huang B, Ying F, Wen Y, et al. EZH2/H3K27Me3 and phosphorylated $\mathrm{EZH} 2$ predict chemotherapy response and prognosis in ovarian cancer. PeerJ. 2020;8:e9052. https://doi.org/10.7717/peerj.9052.

14. Roy DM, Walsh LA, Chan TA. Driver mutations of cancer epigenomes. Protein Cell. 2014;5(4):265-96. https://doi.org/10.1007/s13238-014-0031-6.

15. Dalgliesh GL, Furge K, Greenman C, Chen L, Bignell G, Butler A, et al. Systematic sequencing of renal carcinoma reveals inactivation of histone modifying genes. Nature. 2010:463(7279):360-3. https://doi.org/10.1038/nature08672.

16. Gui Y, Guo G, Huang Y, Hu X, Tang A, Gao S, et al. Frequent mutations of chromatin remodeling genes in transitional cell carcinoma of the bladder. Nat Genet. 2011;43(9):875-8. https://doi.org/10.1038/ng.907.

17. Pereira F, Barbáchano A, Silva J, Bonilla F, Campbell MJ, Muñoz A, et al. $\mathrm{KDM6B} / \mathrm{JMJD3}$ histone demethylase is induced by vitamin D and modulates its effects in colon cancer cells. Hum Mol Genet. 2011;20(23):4655-65. https://doi.org/10.1093/hmg/ddr399.

18. Nassar AH, Umeton R, Kim J, Lundgren K, Harshman L, Van Allen EM, et al. Mutational analysis of 472 Urothelial carcinoma across grades and anatomic sites. Clin Cancer Res. 2019;25(8):2458-70. https://doi.org/10.1158/1078-0432.CCR-18-3147.

19. Hurst CD, Alder O, Platt FM, Droop A, Stead LF, Burns JE, et al. Genomic Subtypes of Non-invasive Bladder Cancer with Distinct Metabolic Profile and Female Gender Bias in KDM6A Mutation Frequency. Cancer Cell. 2017;32(5): 701-715.e707

20. Kaneko S, Li X.X chromosome protects against bladder cancer in females via a KDM6A-dependent epigenetic mechanism. Sci Adv. 2018;4(6):eaar5598.

21. Jansen CS, Prokhnevska N, Master VA, Sanda MG, Carlisle JW, Bilen MA, et al. An intra-tumoral niche maintains and differentiates stem-like CD8 T cells. Nature. 2019:576(7787):465-70. https://doi.org/10.1038/s41586-019-1836-5.

22. Lang A, Yilmaz M, Hader C, Murday S, Kunz X, Wagner N, et al. Contingencies of UTX/KDM6A Action in Urothelial Carcinoma. Cancers (Basel). 2019:11(4):481.

23. Kobatake K, Ikeda Kl, Nakata Y, Yamasaki N, Ueda T, Kanai A, et al. Kdm6a deficiency activates inflammatory pathways, promotes M2 macrophage polarization, and causes bladder Cancer in cooperation with p53 dysfunction. Clin Cancer Res. 2020;26(8):2065-79. https://doi.org/10.1158/1 078-0432.CCR-19-2230.

24. Itoh Y, Golden LC, Itoh N, Matsukawa MA, Ren E, Tse V, et al. The X-linked histone demethylase Kdm6a in CD4+ T lymphocytes modulates autoimmunity. J Clin Invest. 2019;129(9):3852-63. https://doi.org/10.1172/JCI126250.

\section{Publisher's Note}

Springer Nature remains neutral with regard to jurisdictional claims in published maps and institutional affiliations. 